\title{
How to optimize aesthetic outcomes in implant- based breast reconstruction
}

\author{
Maurizio Bruno Nava ${ }^{1,2,3}$, Giuseppe Catanuto ${ }^{3,4}$, Nicola Rocco ${ }^{3,5}$ \\ ${ }^{1}$ Department of Surgery, Valduce Hospital, Como; ${ }^{2}$ Department of Plastic Surgery, University of Milan, Milan; ${ }^{3}$ Group for Reconstructive and \\ Therapeutic Advancements, Milan, Naples, Catania; ${ }^{4}$ Multidisciplinary Breast Unit, Azienda Ospedaliera Cannizzaro, Catania; \\ ${ }^{5}$ Department of Clinical Medicine and Surgery, University of Naples, Naples, Italy
}

The evolution of breast reconstructive surgery keeps pace with the evolution of breast oncologic surgery. The reconstructive choice should always balance the optimal local control of disease and the best cosmetic result, reflecting an informed decision that is shared with the patient, who is always at the center of the decision-making process. Implant-based breast reconstruction following mastectomy represents a complex choice. In order to obtain optimal results, the following considerations are mandatory: thorough preoperative planning, a complete knowledge of the devices that are used, accurately performed surgery, and appropriate follow-up.

Keywords Breast cancer / Breast reconstruction / Breast prosthesis
Correspondence: Nicola Rocco Department of Clinical Medicine and Surgery, University of Naples

"Federico II", Via S. Pansini 5, 80131, Naples, Italy

Tel: +39-3383450357

Fax: +39-0817462515

E-mail: nicolarocco2003@gmail.com

This invited manuscript is based on the topic of Maurizio Bruno Nava's presentation at the 74th Congress of the Korean Society of Plastic and Reconstructive Surgeons on November 20, 2016, in Seoul, Korea.

Received: 11 Mar $2017 \bullet$ Revised: 12 Jun 2017 • Accepted: 22 Jun 2017

pISSN: 2234-6163 • elSSN: 2234-6171 • https://doi.org/10.5999/aps.2017.00528・Arch Plast Surg 2018;45:4-13

\section{INTRODUCTION}

The evolution of breast reconstructive surgery keeps pace with the evolution of breast oncologic surgery.

In the 1980s and early 1990s, the clouds shading the metaphorical hell of Halsted [1] mastectomy parted, revealing the heaven of Fisher lumpectomy $[2,3]$ and Veronesi quadrantectomy [4]. In the late 1990s and early 2000s, the separate worlds of mastectomy and lumpectomy started to work together with the development of oncoplastic breast surgery. Breast reconstruction became a standard treatment, and a tremendous range of progressively less aggressive surgical techniques have been offered to women with a diagnosis of breast cancer, achieving optimal oncologic and reconstructive results regardless of the extent of breast conservation.
In the 2000s, breast cancer surgery came to no longer represent a dichotomous choice [5]. The higher sensitivity of diagnostic imaging, new genetic studies, and new opportunities for risk-reducing procedures led to a renewed increase in the mastectomy rate during the first decade of the 2000s [6,7]. A higher percentage of women well informed about the equivalence in terms of survival between breast-conserving surgery and mastectomy came to prefer mastectomy thanks to the excellent aesthetic results obtained with reconstruction [8].

Currently, oncologic breast surgery represents a decision that is shared with the patient, with the surgical treatment tailored to fit each patient, never forgetting the patient's own desires and wishes.

The oncoplastic decision-making process should balance the optimal local control of disease with the best reconstructive re- 
sult, while providing all the necessary information to the patient in order to make a shared decision, with the patient always at the center of the decision-making process $[9,10]$. In order to achieve this goal, each surgeon involved in the oncoplastic decisionmaking process should be aware of all possible breast reconstructive options. Even though a single surgeon may not be able to perform each surgical technique (the so-called and much-desired 'vertical surgeon'), he or she must at least be aware of all techniques and indications to allow the patient to make the bestinformed choice for her breast reconstruction.

The years from the 1980s to the first decade of the 21st century saw an amazing evolution of biomaterials and devices available to the breast surgeon; for instance, round implants, with a smooth surface and filled with low-cohesion gel, have given way to anatomical implants, with a textured surface and filled with a high-cohesion gel. The evolution of biomaterials led to a decreased rate of capsular contracture around implants, with significant improvements in the reconstructed breast shape, paving the way for new reconstructive paradigms [11].

The evidence in the literature has definitively demonstrated that immediate breast reconstruction following mastectomy does not affect oncologic outcomes, even in patients with advanced disease, and does not significantly delay adjuvant therapies $[12,13]$.

Moreover, as we demonstrated more than 25 years ago, immediate breast reconstruction presents great advantages from a psychological point of view, while delayed breast reconstruction can trigger anxiety and distress during the period of mastectomy in women and their partners [14].

In 2008, we presented an alternative surgical model for breast reconstruction using extra-projected implants, achieving a wellshaped breast and contralateral reshaping with less surgical trauma, reserving autologous flap reconstruction only for patients treated with radiotherapy [15]. The 1990s paradigm, in which large breasts are reconstructed with an autologous flap, medium/small breasts are reconstructed with an implant, and the contralateral breast may not undergo reconstruction at all, was subverted by the extra-projected implants surgical model.

We also demonstrated that contralateral breast symmetrization with pexy-reduction techniques does not significantly impact oncologic follow-up in breast cancer patients; in particular, these techniques do not lead to a significantly higher need for secondlevel imaging exams and diagnostic biopsies, and therefore do not delay the diagnosis of cancer in the contralateral breast [16].

We also developed tools for determining the optimal timing of radiotherapy in implant-based breast reconstruction in the form of our decision algorithms, which are a very useful tool for the breast surgeon to plan together with his or her patient the best reconstructive option after radiotherapy or in anticipation of post-mastectomy radiotherapy [17].

The first decade of the new century also saw the possibility of treating radiotherapy damage with autologous fat cell transplantation, which is a fast and safe procedure that has further extended the indications for extra-projected implant-based reconstruction [18].

We rationalized and systematically organized our reconstructive algorithms, shedding new light on so-called "conservative mastectomies" [19-21] and expanding breast reconstructive opportunities and choices with the introduction of the skin-reducing mastectomy for patients with large and ptotic breasts [22-24].

Implant-based breast reconstruction following mastectomy represents a complex choice. In order to obtain optimal results, the following considerations are mandatory: thorough preoperative planning, a complete knowledge of the devices that are used, accurately performed surgery, and appropriate follow-up.

We must always remember that a good cosmetic result in breast reconstructive surgery starts with a good mastectomy. We now know that total preservation of the breast envelope is possible in patients with early-stage breast cancer without clinical involvement of the nipple-areolar complex (NAC). The preservation of the whole breast envelope is more of a technical challenge than an oncologic issue, as long mastectomy skin flaps only receive blood supply from the subdermal plexus. The risk factors for ischemic complications (previous radiotherapy, smoking, etc.) should be thoroughly assessed preoperatively, and patients at high risk should be discouraged from undergoing nipple preservation. The larger the breast is, the higher is the ischemic complication rate; large breast envelopes cannot be easily preserved, both due to the risk of necrosis and the possibility of dislocation of the NAC. We consider nipple preservation in cases with a nipple-to-sternal notch distance less than or equal to $25 \mathrm{~cm}$. In such cases, we opt for a nipple-sparing skin reducing technique, leaving the NAC on a superior pedicle. In patients with a nipple-to-sternal notch distance longer than $25 \mathrm{~cm}$, a Wise pattern skin-reducing mastectomy with synchronous reconstruction of the NAC can be performed. Fig. 1 [25-27] contains a flow chart providing guidance for the best surgical choice in light of risk factors for NAC loss and poor cosmetic outcomes.

Women with early-stage breast cancer for whom a mastectomy is planned can experience good outcomes with either a 2- or a 1-stage implant-based reconstruction if accurate planning is performed. We can consider 3 categories of breast morphology, assuming that the risk of necrotic complications has been preoperatively estimated to be low: (1) patients with small and very small breasts; (2) patients with medium-sized and/or moderately ptotic breasts; (3) patients with large and ptotic breasts. 
Fig. 1. Decision-making algorithm for NAC-sparing mastectomies

NAC, nipple-areolar complex.

A proposed algorithm to extend the indications to NAC preservation
Risk of necrosis and no risk of cosmetic failure

Risk of cosmetic failure and no risk of necrosis

Risk of necrosis and risk of cosmetic failure

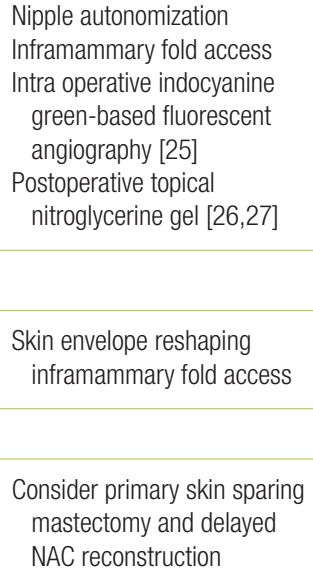

Nipple autonomization Inframammary fold access Intra operative indocyanine green-based fluorescent angiography [25] Postoperative topical nitroglycerine gel $[26,27]$

Skin envelope reshaping inframammary fold access

Consider primary skin sparing mastectomy and delayed NAC reconstruction

\section{PATIENTS WITH SMALL AND VERY SMALL BREASTS}

Low-risk patients with small breasts can be offered a direct-toimplant (DTI) nipple-sparing mastectomy with acellular dermal matrix (ADM) use and contralateral augmentation. On the mastectomy side, the implant width should be selected according to the breast footprint, subtracting the thickness of the skin flaps and adding $1 \mathrm{~cm}$. The height of the implant is calculated according to the height of the contralateral breast. In such patients, we suggest using extra-projected or full-projected implants when the NAC is preserved. The contralateral implant will have the same width of the mastectomy-side implant, but reduced by approximately the thickness of the skin-glandular flap (Fig. 2).

\section{PATIENTS WITH MEDIUM-SIZED AND/ORMODERATELY PTOTIC BREASTS}

These patients can undergo a DTI breast reconstruction without contralateral adjustment. Even in such cases, we must assess the footprint of the breast. We suggest avoiding extra-projected implants, especially in reconstructions of slightly ptotic breasts that may be treated with full- or medium-projected implants (Fig. 3).

\section{Fig. 2. Implant selection guide for small breasts}

Implant selection guide for DTI breast reconstruction in women with small and very small breasts. DTI, direct-to-implant; C/L, con-

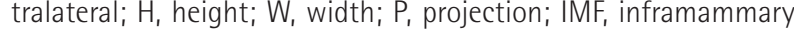
fold; $\mathrm{SN}-\mathrm{N}$, sternal notch to nipple distance.

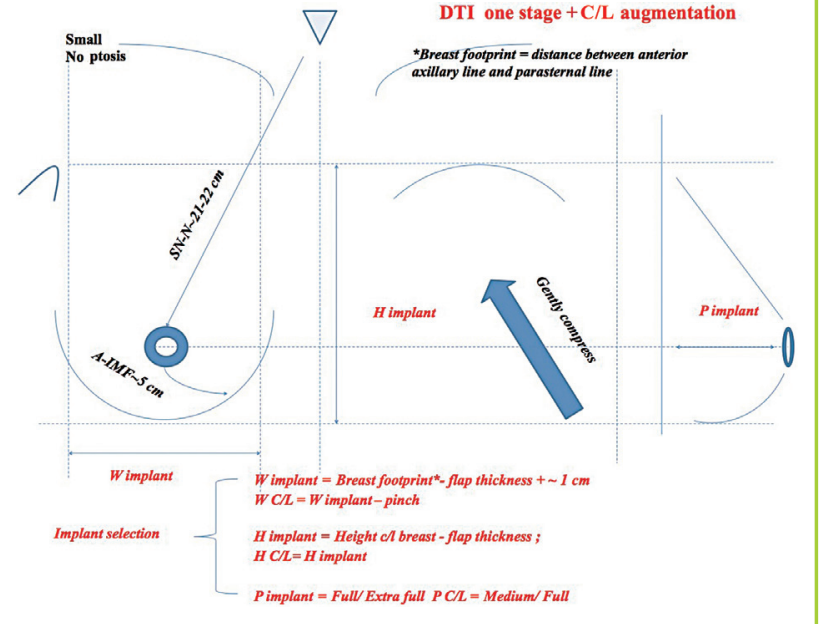

\section{Fig. 3. Implant selection guide for medium breasts}

Implant selection guide for DTI breast reconstruction in women with medium-sized and/or moderately ptotic breasts. DTI, direct-toimplant; $C / L$, contralateral; $H$, height; $W$, width; $P$, projection; IMF, inframammary fold; $\mathrm{SN}-\mathrm{N}$, sternal notch to nipple distance.

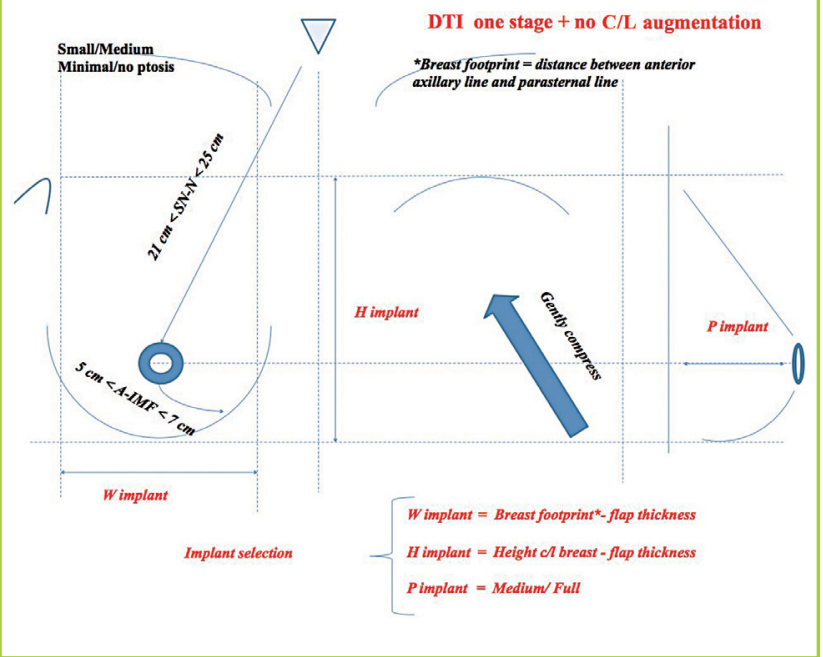

\section{PATIENTS WITH LARGE AND PTOTIC BREASTS}

In such cases, we recommend a DTI breast reconstruction that can be performed using a Wise pattern access with or without nipple preservation (depending on the nipple-to-sternal notch distance) and contralateral breast reduction or mastopexy. The implant choice follows the same rules already considered for the 
other subgroups. In the assessment of the footprint, we advise avoiding implants exceeding the anterior axillary line, even if the breast boundaries would overcome this border (Fig. 4).

All other cases, including medium-sized ptotic breasts and very large and ptotic breasts, can be treated using a 2-stage approach. We also offer this reconstructive option to patients who are at a higher risk of complications and to those who could benefit from reduced skin tension and viable coverage of the implant. The implant selection for 2 -stage reconstructions starts with an assessment of the breast footprint for the selection of the tissue expander. The final implant on the reconstructive side may sometimes exceed the original width of the tissue expander by 0.5 to $1 \mathrm{~cm}$. This may be due to the effects of the capsulectomy, which may create an enlarged pocket at the second stage. Contralateral adjustments may be more frequently required in

\section{Fig. 4. Implant selection guide for large breasts}

Implant selection guide for DTI breast reconstruction in women with large and ptotic breasts. DTI, direct-to-implant; C/L, contralat-

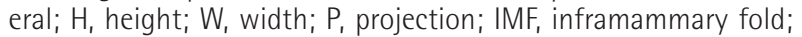
$\mathrm{SN}-\mathrm{N}$, sternal notch to nipple distance.

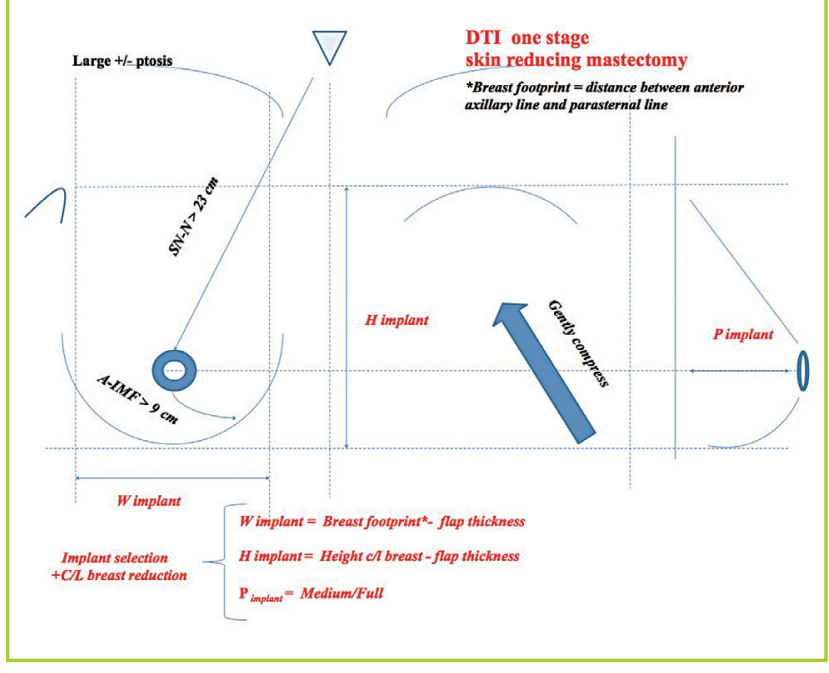

2-stage approaches than in DTI reconstructions.

When planning and performing a breast reconstruction, we must always consider the inferior pole, the inframammary fold, the superior slope, and the breast projection. In particular, we have described how to perfectly rebuild the inframammary fold, using a running suture at the level of the superficial fascial system [28].

Preoperative digital mammography is a very useful tool for the breast surgeon in order to plan the best reconstructive option following mastectomy. Digital mammography can clearly determine the thickness of breast tissue coverage overlying the gland, corresponding to the superficial tissues between the skin and the Cooper ligaments (i.e., the dermis and subcutaneous fat), thus facilitating the planning of the optimal reconstructive technique. We recently proposed a breast tissue coverage classification (BTCC) according to the thickness of the superficial tissues covering the mammary gland, as measured in centimeters: type 1 , poor coverage, $<1 \mathrm{~cm}$; type 2 , medium coverage, $1-2 \mathrm{~cm}$; type 3 , good coverage, $>2 \mathrm{~cm}$ [29]. According to the BTCC, we suggest 2 -stage implant-based breast reconstruction followed by autologous fat tissue transplantation before the second stage for type 1 patients, 1-stage implant-based breast reconstruction with ADM (Fig. 5) or synthetic meshes (with or without considering autologous fat tissue transplantation) (Fig. 6) for type 2 patients, and 1-stage implant-based breast reconstruction with synthetic mesh use for type 3 patients (Fig. 7).

Our target and main aim for the next years, through 2020, is to further reduce surgical aggressiveness, improving cosmetic and reconstructive outcomes. Our path of research and development will focus on advances in biomaterials, with new and safer ADMs (Fig. 8) and synthetic meshes together with enhanced fat grafting, in order to achieve the next step, the 'hybrid reconstruction,' which will allow immediate breast reconstruction combining the use of fat, implants, and meshes.

The so-called 'hybrid' model will represent a sort of evolution

\section{Fig. 5. Right nipple-areolar complex-sparing mastectomy}

Direct-to-implant reconstruction with acellular dermal matrix and Allergan Style $410 \mathrm{MX}$ implant $(370 \mathrm{~mL})$. Contralateral breast augmentation (Allergan Style 410 MF implant, $225 \mathrm{~mL}$ ). (A) Postoperative view at a 2-year follow-up. (B) Preoperative digital mammography showing a type 2 breast. RMLO, right medio lateral oblique; LMLO, left medio lateral oblique.
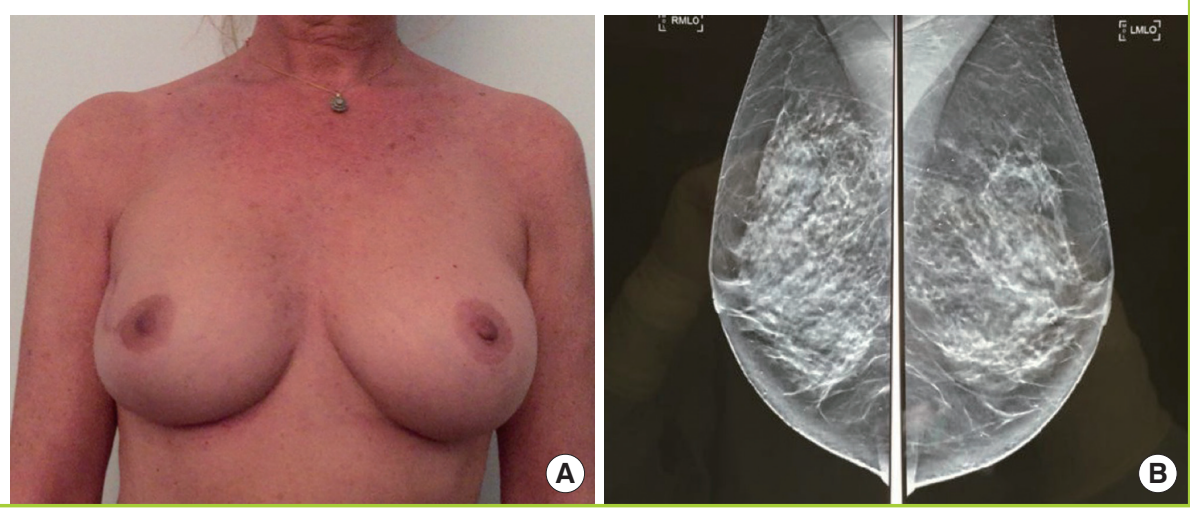


\section{Fig. 6. Left NAC-sparing mastectomy}

Direct-to-implant reconstruction with synthetic mesh and an Allergan Style 510 MX implant (width, $13.5 \mathrm{~cm}$; height, $12.5 \mathrm{~cm}$; projection, $5.9 \mathrm{~cm}$; $385 \mathrm{~mL}$ ). (A) Preoperative view. (B) Preoperative digital mammography showing a type 2 breast. (C) Postoperative view at a 2-year follow-up. NAC, nipple-areolar complex.
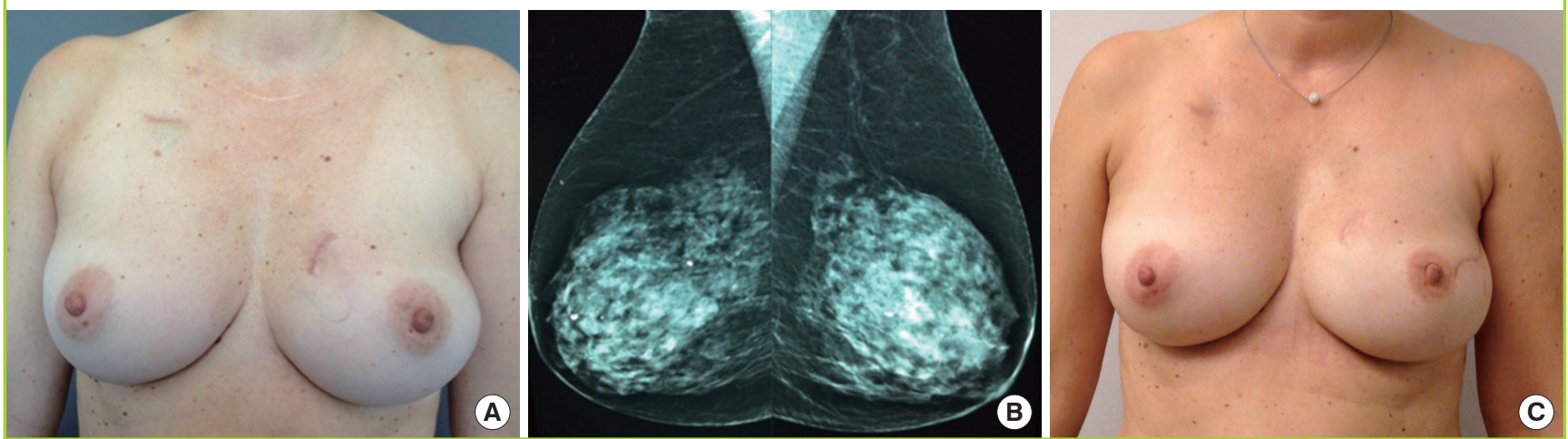

Fig. 7. Bilateral NAC-sparing mastectomy

Direct-to-implant reconstruction with titanium-coated polypropylene mesh (TiLOOP Bra) (pfm medical, Cologne, Germany) and an Allergan Style 410 MX implant (width, $13 \mathrm{~cm}$; height, $12.1 \mathrm{~cm}$; projection, $6.1 \mathrm{~cm} ; 410 \mathrm{~mL})$. (A) Preoperative digital mammography showing a type 3 breast. (B) Results at 2 years. NAC, nipple-areolar complex.
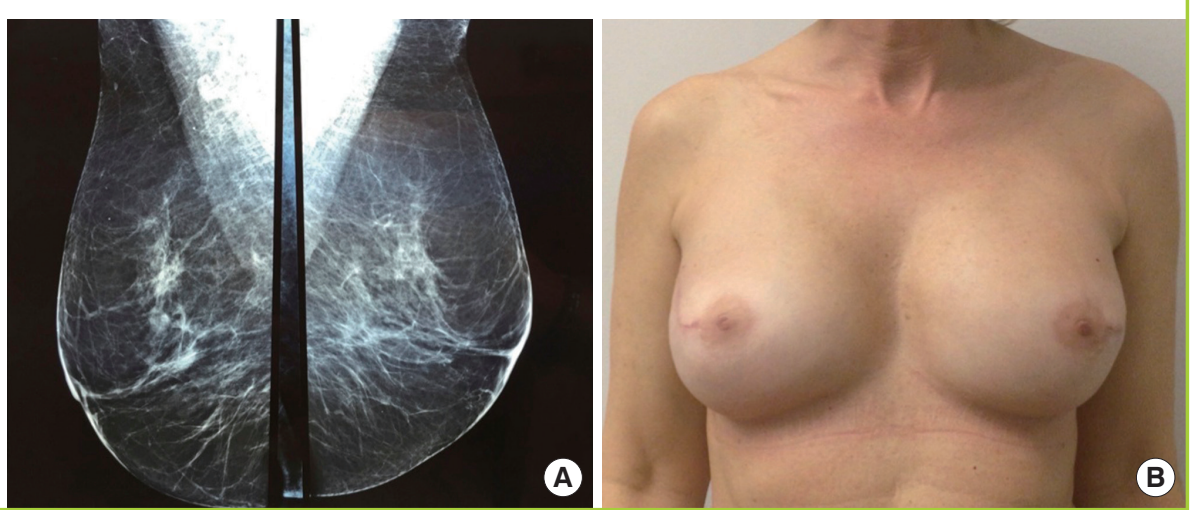

\section{Fig. 8. Bilateral NAC-sparing mastectomy}

Direct-to-implant reconstruction with an Allergan Style $410 \mathrm{MF}$ implants (295 mL) and acellular dermal matrix (ARTIA, LifeCell Corp, Branchburg, NJ, USA). (A) Preoperative markings. (B) Immediate postoperative results. (C) Postoperative results at a 6-month follow-up. NAC, nipple-areolar complex.
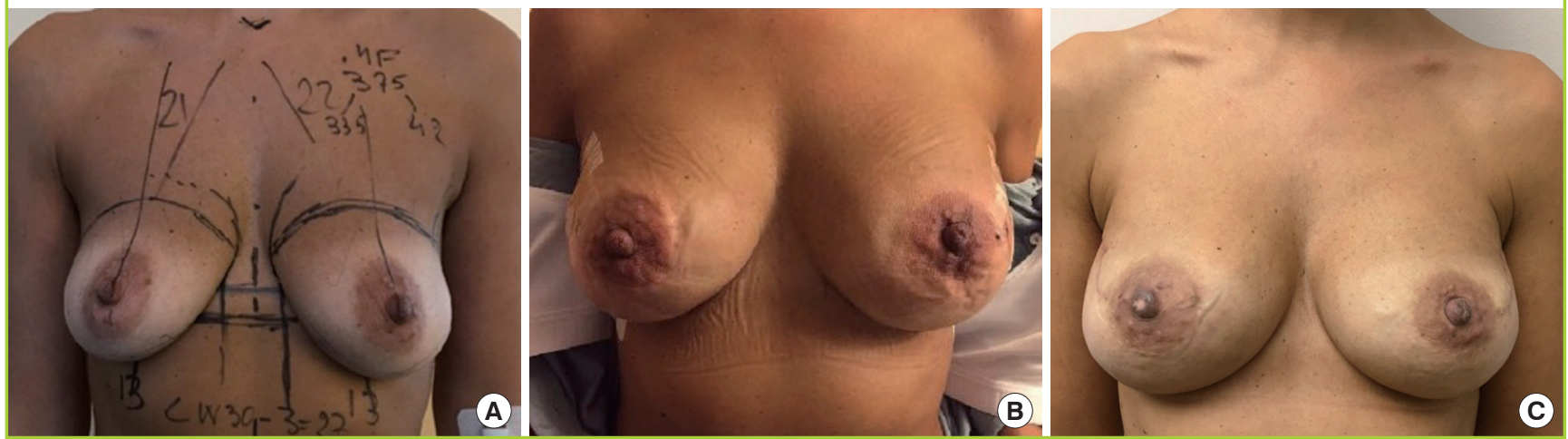

of the surgical model of extra-projected implants, allowing greater stability of the results (in ptotic breasts as well), a stronger aesthetic impact (we add body recontouring to the mediumsize extra-projected reconstructive model, transferring fat cells from other anatomical body areas to the breast), and better feel- ing and perception of the implant (also reducing capsular contracture). The development of fat-injection techniques to refine the results of implant-based breast reconstructions is changing the outcomes of breast surgery [30].

We are firmly convinced that the future of breast reconstruc- 


\section{Fig. 9. Hybrid breast reconstruction}

Hybrid breast reconstruction decision-making algorithm for high-BMI patients. BMI, body mass index; C/L, contralateral; XP, extra-projected.

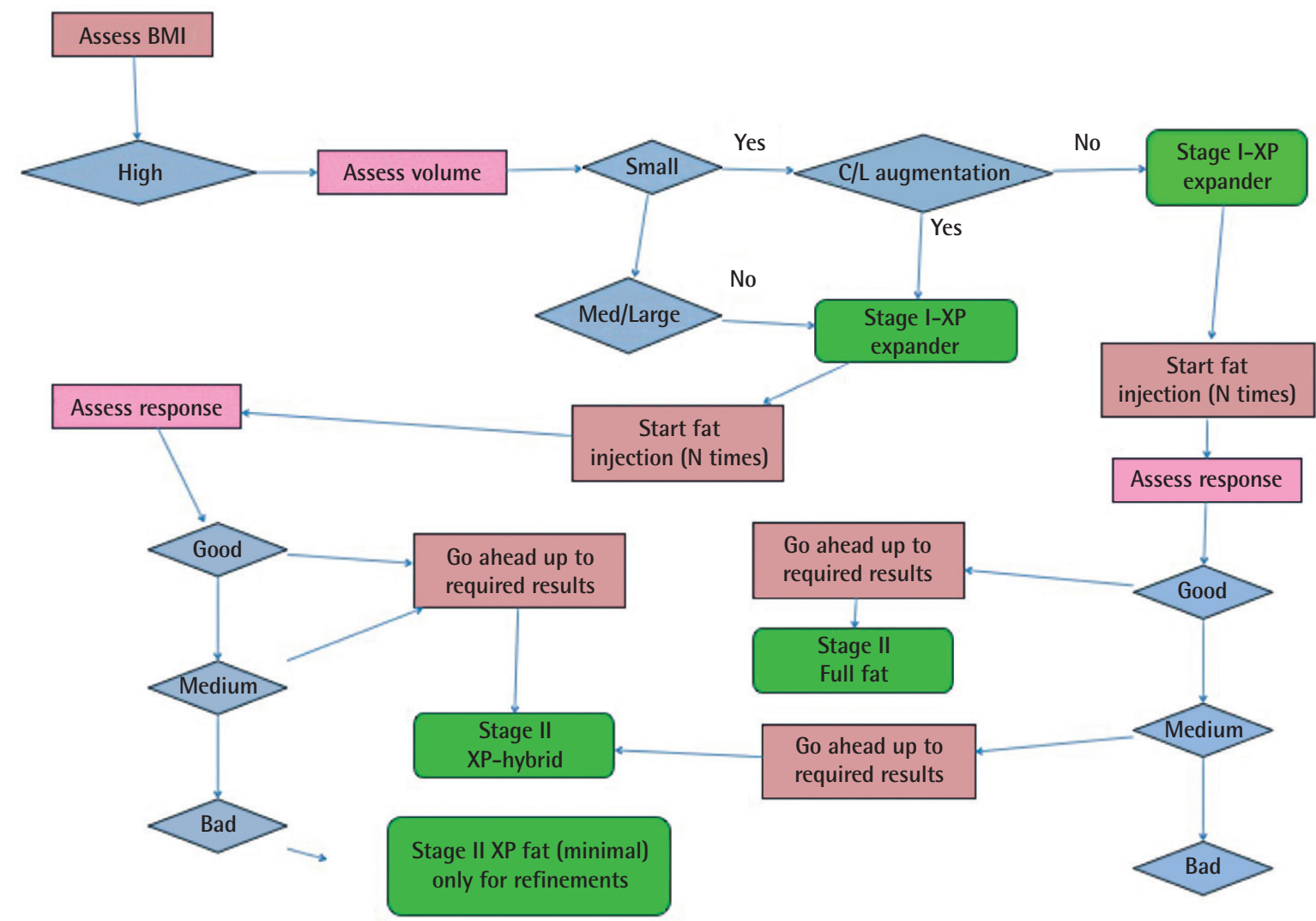

tion will be based on refined applications of autologous fat grafting (AFG), particularly in light of the growing, although not definitive, evidence of the safety of AFG use in breast reconstruction following mastectomy for breast cancer [31]. This is the reason why we are currently developing and validating an innovative surgical model that we have termed hybrid breast reconstruction. This new reconstructive system encompasses prostheses and fat grafting as integrated tools to improve the final reconstructive outcome. In our view, fat grafting should not only be employed for breast reconstruction refinements or to treat radio-induced tissue damage, but should be a preoperatively planned part of the reconstruction. The final volume we want to achieve will be made up of fat and implants in a variable percentage according to the patient's body contour and breast morphology. Hybrid breast reconstruction will represent the best option for patients with medium-large breasts with moderate-high ptosis: they will benefit from the positioning of an expander (in immediate or delayed reconstruction) together with a first round of fat grafting to the reconstructed breast, followed by at least 2 other lipotransfers (the number of procedures will be tailored to each patient according to body contour and the desired final breast size), while progressively deflating the expander. At the end of the fat/expander-mediated tissue expansion, the patient will undergo placement of a standard expander for permanent implant replacement, following a different time protocol, with the second reconstruction time planned in a shorter period than is customary for a usual 2-stage reconstruction. The decision to pursue the hybrid reconstructive pathway must be shared with the patient, who must be adequately motivated to pursue the entire planned reconstructive protocol, which consists of multiple, although minimally invasive, procedures. Women who undergo hybrid reconstructions will benefit from the positioning of a smaller permanent implant compared with a standard 2 -stage reconstruction, with better outcomes both regarding surgical technical aspects (fewer immediate and long-term complications) and their quality of life (more natural feeling of the implant). We schematized the hybrid reconstructive approach in 3 algorithms according to the body mass index of the patient to be treated (Figs. 9-11). Patients with small or very large breasts with high ptosis are good candidates for a 1-stage directto-implant reconstruction, following a conservative mastectomy (a skin/NAC-sparing and a skin reducing mastectomy, respec- 


\section{Fig. 10. Hybrid breast reconstruction}

Hybrid breast reconstruction decision-making algorithm for medium-BMI patients. BMI, body mass index; $\mathrm{XP}$, extra-projected.

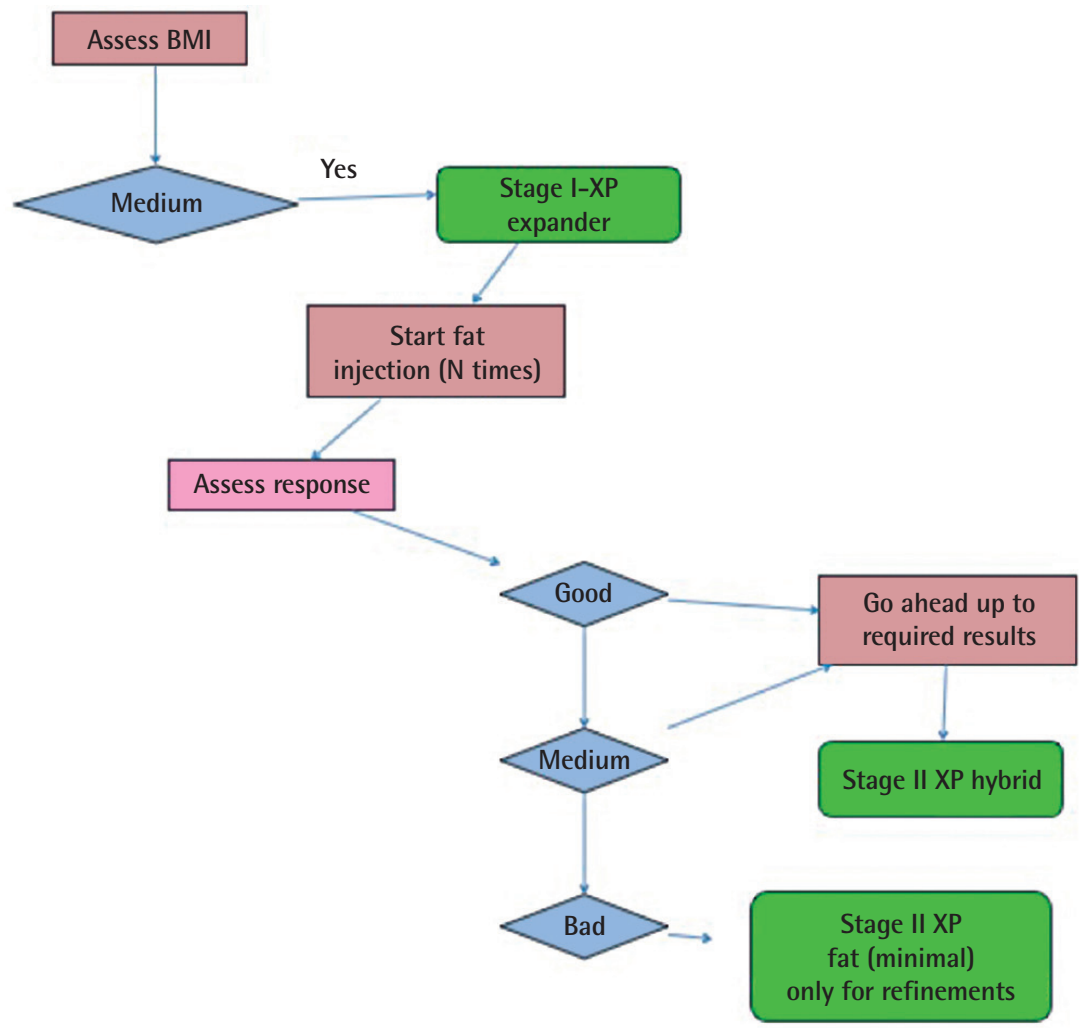

tively). These patients also benefit from planned fat grafting, after the main (permanent implant positioning) procedure, in order to achieve optimal cosmetic results. This is another option for a planned hybrid reconstruction program: placement of a permanent implant smaller than the one usually considered for a 1-stage reconstruction, with the final breast volume and shape reached with a planned lipotransfer. In this way, breast reconstructions will more and more closely reproduce the results and outcomes of cosmetic breast augmentation. We have used the hybrid breast reconstruction technique in more than 350 reconstructions, both immediate and delayed, 2-stage and DTI, without experiencing higher loco-regional recurrence or complication rates when compared with conventional 2-stage reconstructions, and have obtained excellent aesthetic results and high patient satisfaction levels (Fig. 12). We think that the total replacement of prostheses with fat would be desirable, but is not realistic at the moment.

The use of implants will continue to be necessary unless new technologies are discovered to stabilize the shape of full-fat reconstructed breasts, achieving better fat survival through fat enhancement techniques [32] or through dedicated scaffolds to drive cell regeneration and shape definition. We are also exploring this field in a preclinical setting, as presented in a recent paper [33]. We presented an innovative breast reconstructive technique, with the use of a reverse-engineering approach and additive manufacturing methods (3-dimensional [3D] image capture, 3D modeling, and rapid prototyping with 3D printers) combined with autologous fat grafting. Ultimately, a 3D structure with autologous adipose-derived stem cells will be used as a construct for tissue regeneration. The 3D-printed scaffold will be generated from computed tomography or magnetic resonance imaging data of the breast that will be reconstructed through image capture and analysis techniques, and will be placed in the subcutaneous position at the level of the mastectomy side. The scaffold will be colonized with autologous fat tissue over the course of multiple sessions. The biodegradable customized structure will help to maintain the breast shape, and a natural consistency will be obtained via fat grafting, with additional consideration of adequate enhancement techniques.

Future clinical studies will validate the use of these innovative breast reconstructive techniques, which will likely become standard options for next-generation breast surgeons. 


\section{Fig. 11. Hybrid breast reconstruction}

Hybrid breast reconstruction decision-making algorithm for low-BMI patients. BMI, body mass index; XP, extra-projected.

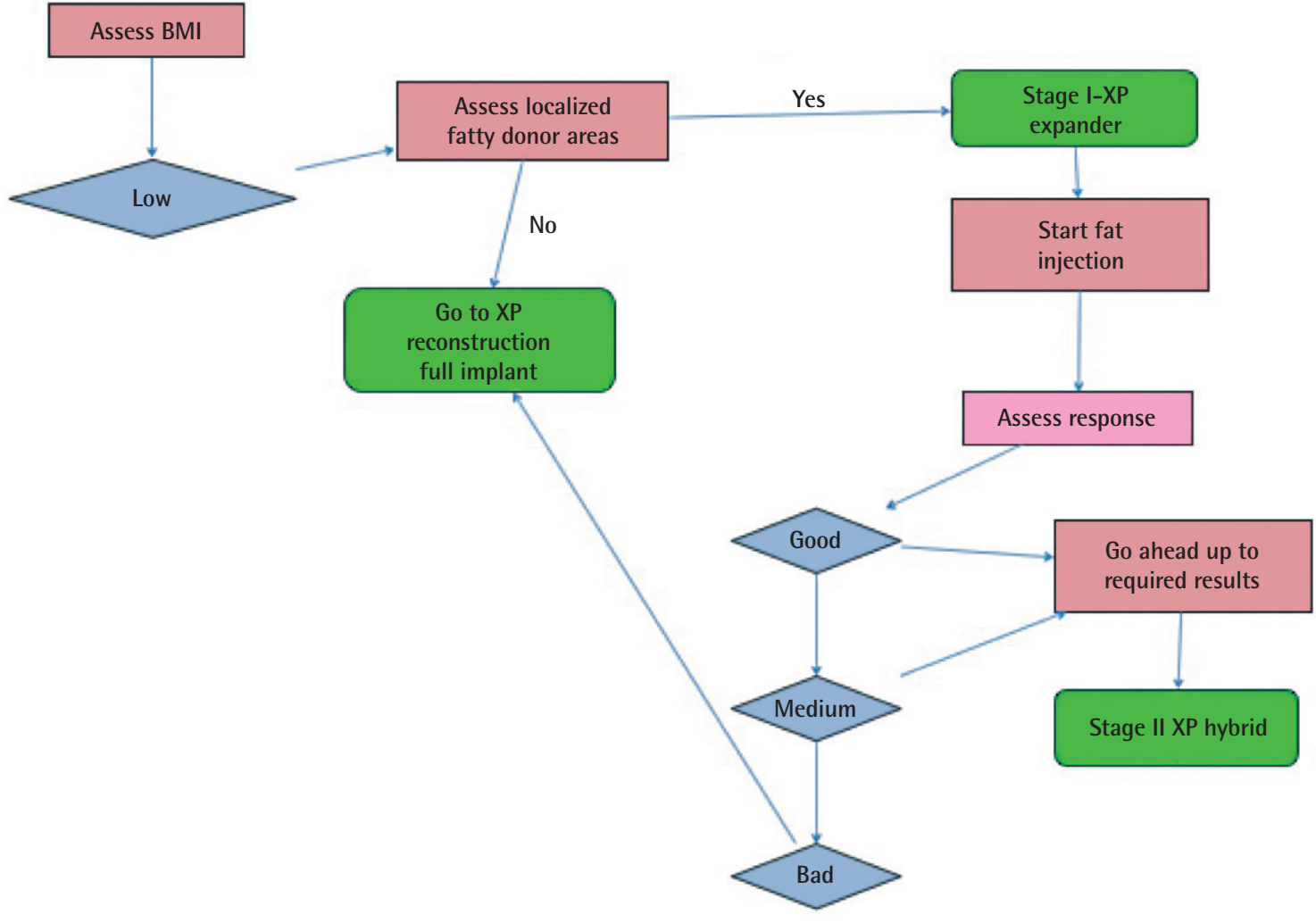

\section{Fig. 12. Hybrid breast reconstruction}

Postoperative results at a 5-year followup. The patient underwent a right modified radical mastectomy and 2-stage reconstruction. Three bilateral fat transfer sessions were performed between the first stage and the second stage (Allergan Style 410 MF $335 \mathrm{~mL}$ implant), while progressively deflating the expander with a definitive fat-transfer volume of $250 \mathrm{~mL}$ for each breast. Left symmetrization augmentation mastopexy (Allergan Style 410 MM $215 \mathrm{~mL}$ implant).
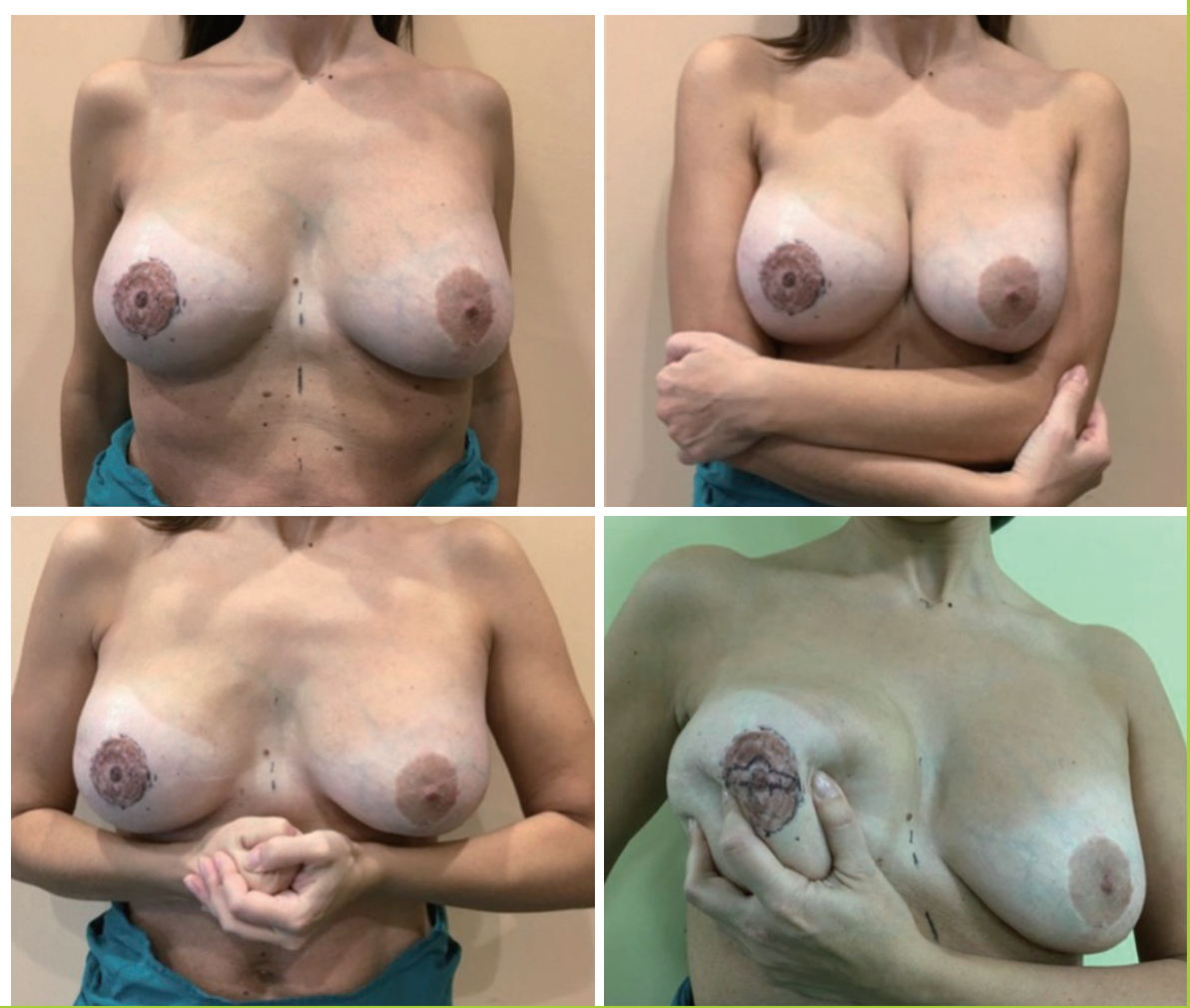


\section{CONFLICT OF INTEREST}

No potential conflict of interest relevant to this article was reported.

\section{PATIENT CONSENT}

The patient provided written informed consent for the publication and the use of their images.

\section{REFERENCES}

1. Halsted WS. I. The results of operations for the cure of cancer of the breast performed at the Johns Hopkins Hospital from June, 1889, to January, 1894. Ann Surg 1894;20:497555.

2. Fisher B, Bauer M, Margolese R, et al. Five-year results of a randomized clinical trial comparing total mastectomy and segmental mastectomy with or without radiation in the treatment of breast cancer. N Engl J Med 1985;312:665-73.

3. Fisher B, Redmond C, Poisson R, et al. Eight-year results of a randomized clinical trial comparing total mastectomy and lumpectomy with or without irradiation in the treatment of breast cancer. N Engl J Med 1989;320:822-8.

4. Veronesi U, Saccozzi R, Del Vecchio M, et al. Comparing radical mastectomy with quadrantectomy, axillary dissection, and radiotherapy in patients with small cancers of the breast. N Engl J Med 1981;305:6-11.

5. della Rovere GQ Benson JR, Breach N, et al. Oncoplastic and reconstructive surgery of the breast. London: Taylor \& Francis; 2004.

6. Katipamula R, Degnim AC, Hoskin T, et al. Trends in mastectomy rates at the Mayo Clinic Rochester: effect of surgical year and preoperative magnetic resonance imaging. J Clin Oncol 2009;27:4082-8.

7. McGuire KP, Santillan AA, Kaur P, et al. Are mastectomies on the rise? A 13-year trend analysis of the selection of mastectomy versus breast conservation therapy in 5,865 patients. Ann Surg Oncol 2009; 16:2682-90.

8. Bhat S, Orucevic A, Woody C, et al. Evolving trends and influencing factors in mastectomy decisions. Am Surg 2017; 83:233-8.

9. Catanuto G, Rocco N, Nava MB. Surgical decision making in conservative mastectomies. Gland Surg 2016;5:69-74.

10. Catanuto G, Pappalardo F, Rocco N, et al. Formal analysis of the surgical pathway and development of a new software tool to assist surgeons in the decision making in primary breast surgery. Breast 2016;29:74-81.
11. Rocco N, Rispoli C, Moja L, et al. Different types of implants for reconstructive breast surgery. Cochrane Database Syst Rev 2016;(5):CD010895.

12. Zhang P, Li CZ, Wu CT, et al. Comparison of immediate breast reconstruction after mastectomy and mastectomy alone for breast cancer: a meta-analysis. Eur J Surg Oncol 2017;43:285-93.

13. Park SH, Han W, Yoo TK, et al. oncologic safety of immediate breast reconstruction for invasive breast cancer patients: a matched case control study. J Breast Cancer 2016;19:6875.

14. Filiberti A, Rimoldi A, Callegari M, et al. Immediate versus delayed breast reconstruction A psychological answer. Eur J Plast Surg 1990;13:55-8.

15. Nava MB, Spano A, Cadenelli P, et al. Extra-projected implants as an alternative surgical model for breast reconstruction: implantation strategy and early results. Breast 2008; 17 : 361-6.

16. Nava MB, Rocco N, Catanuto G, et al. Impact of contra-lateral breast reshaping on mammographic surveillance in women undergoing breast reconstruction following mastectomy for breast cancer. Breast 2015;24:434-9.

17. Nava MB, Pennati AE, Lozza L, et al. Outcome of different timings of radiotherapy in implant-based breast reconstructions. Plast Reconstr Surg 2011;128:353-9.

18. Nava MB, Catanuto G, Pennati AE, et al. Lack of activation of telomere maintenance mechanisms in human adipose stromal cells derived from fatty portion of lipoaspirates. Plast Reconstr Surg 2015;135:114e-123e.

19. Nava MB, Catanuto G, Pennati A, et al. Conservative mastectomies. Aesthetic Plast Surg 2009;33:681-6.

20. Nava MB, Rocco N, Catanuto G. Conservative mastectomies: an overview. Gland Surg 2015;4:463-6.

21. Rocco N, Catanuto G, Nava MB. What is the evidence behind conservative mastectomies? Gland Surg 2015;4:50618.

22. Nava MB, Cortinovis U, Ottolenghi J, et al. Skin-reducing mastectomy. Plast Reconstr Surg 2006;118:603-10.

23. della Rovere GQ, Nava M, Bonomi R, et al. Skin-reducing mastectomy with breast reconstruction and sub-pectoral implants. J Plast Reconstr Aesthet Surg 2008;61:1303-8.

24. Nava MB, Ottolenghi J, Pennati A, et al. Skin/nipple sparing mastectomies and implant-based breast reconstruction in patients with large and ptotic breast: oncological and reconstructive results. Breast 2012;21:267-71.

25. Griffiths M, Chae MP, Rozen WM. Indocyanine greenbased fluorescent angiography in breast reconstruction. Gland Surg 2016;5:133-49. 
26. Nahabedian MY. Implant-based breast reconstruction: strategies to achieve optimal outcomes and minimize complications. J Surg Oncol 2016;113:895-905.

27. Dent BL, Small K, Swistel A, et al. Nipple-areolar complex ischemia after nipple-sparing mastectomy with immediate implant-based reconstruction: risk factors and the success of conservative treatment. Aesthet Surg J 2014;34:560-70.

28. Nava M, Quattrone P, Riggio E. Focus on the breast fascial system: a new approach for inframammary fold reconstruction. Plast Reconstr Surg 1998;102:1034-45.

29. Rancati A, Angrigiani C, Hammond D, et al. Preoperative digital mammography imaging in conservative mastectomy and immediate reconstruction. Gland Surg 2016;5:9-14.
30. Zhou Y, Wang J, Li H, et al. Efficacy and safety of cell-assisted lipotransfer: a systematic review and meta-analysis. Plast Reconstr Surg 2016;137:44e-57e.

31. Kronowitz SJ, Mandujano CC, Liu J, et al. Lipofilling of the breast does not increase the risk of recurrence of breast cancer: a matched controlled study. Plast Reconstr Surg 2016; 137:385-93.

32. Tissiani LA, Alonso N. A prospective and controlled clinical trial on stromal vascular fraction enriched fat grafts in secondary breast reconstruction. Stem Cells Int 2016;2016:2636454.

33. Rocco N, Gloria A, De Santis R, et al. Improving outcomes in breast reconstruction: from implant-based techniques towards tissue regeneration. Procedia CIRP 2016;49:23-7. 